\title{
A STATISTICAL APPROACH TO FINGER-PRINT RUN-OF-MINE DIAMONDS USING FTIR SPECTRA, SIZE DISTRIBUTIONS AND PHYSICAL CHARACTERISTICS.
}

\author{
Eva Anckar ${ }^{1}$, John Gurney ${ }^{1}$ and Christien Thiart ${ }^{2}$ \\ 1) Dept. Geological Sciences, University of Cape Town, South Africa \\ 2) Dept. Statistical Sciences, University of Cape Town, South Africa
}

\section{INTRODUCTION}

It can be concluded that although diamond populations from known localities vary widely in their size distribution, proportions of shapes, colours and surface appearance certain diamond characteristics can be considered key features. In several cases such characteristic features can be associated with a specific source (fig 1).
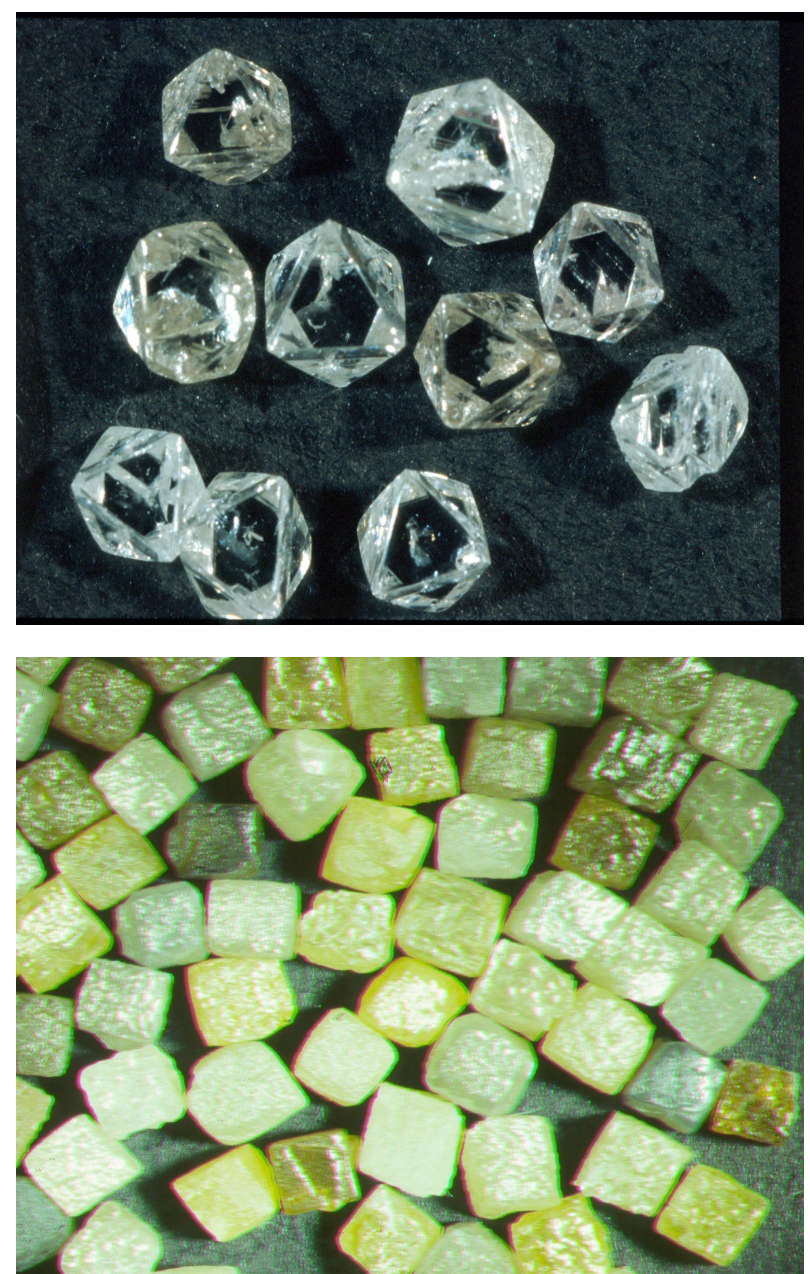

Figure 1. Above - Perfect octahedral crystals with flat faces for characteristic populations at Mir, Russia. Below - Opaque cubic diamond crystals characteristic for Mbuyi Mayi, Zaire.
Added to this, Infra Red spectral patterns also differ from one source to another. Fourier Transform Infrared spectroscopy (FTIR) is a fast and inexpensive technique that performs non-destructive diamond analysis that can yield fundamental information about the environment in which the diamond grew (fig 2), and about the light elements such as oxygen, hydrogen and nitrogen that it has incorporated into its structure. Despite this, on the basis of a detailed assessment of how, when and where diamonds form, it is considered unlikely that individual diamonds can be fingerprinted, except in exceptional cases. Profiling a parcel of diamonds in contrast looks achievable.

\section{PREVIOUS WORK}

Various approaches have been suggested. These include neutron activation analysis and laser ablation ICPMS analysis, both analytical techniques which attempt to determine the trace element levels in the diamond. Neither of these methods shows great promise. Problems start with the fact that the purer (that is more valuable) diamonds yield very little information with these analytical techniques. Such impurities as there are in good quality diamonds are primarily light elements such as oxygen, hydrogen and nitrogen which cannot be determined by these two analytical techniques. It is our experience that these two techniques produce very few measurements above the detection limits of any of the elements in the periodic table and that these measurements are not identifiably diagnostic. In addition, both techniques are destructive as far as gem quality diamonds are concerned; prolonged neutron activation makes the diamonds black throughout and laser ablation ICPMS drills a hole in the diamond which necessarily gets bigger if you want to push the analytical technique to lower detection limits. Such methods therefore have severe limitations on their usefulness to routine investigations. 


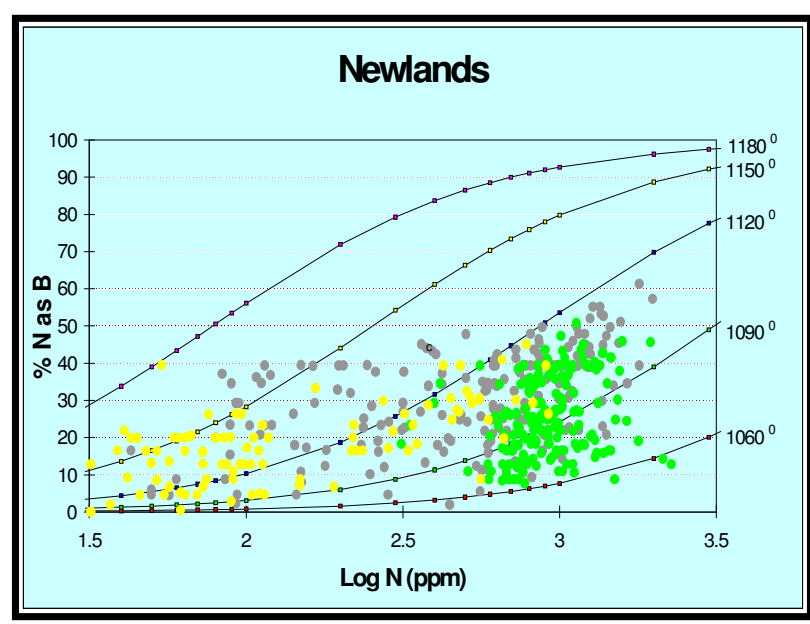

Figure 2. Diamond paragenesis correlates with the relationship between $\mathrm{N}$ and $\% \mathrm{NasB}$ in a study of a suite of diamonds from Newlands, South Africa. (Menzies, 2001) (Green markers represent eclogitic diamonds and peridotitc diamonds are yellow in the diagram)

\section{THIS STUDY}

In this study we utilise the two techniques, FTIR and description of physical properties, in combination, and apply multivariate statistics on the resulting data set consisting of nitrogen content, nitrogen aggregation state, hydrogen content, presence of carbonate, platelet peak strength and position. Additional variables relate to the physical properties of diamonds such as color shape, surface features, cathodoluminescence patterns, size and deformation. Cathodoluminescence is very successful at differentiating between diamonds that have formed in one simple process, and diamonds that have had a more complicated growth history. Variation in color and intensity indicate differences in the amount of impurities such as nitrogen, boron and hydrogen. In this study approximately 2000 records of data are stored in an Access database and form a world library of diamond characteristics.

\section{METHODS}

\section{$4.1 \mathrm{FTIR}$}

FTIR spectra were measured at the University of Cape Town using a Nicolet Magna-IR 560 spectrometer, with an attached $\mathrm{KBr}$ beam splitter and a MCT/A detector. Spectra were recorded over the range $4000-650 \mathrm{~cm}^{-1}$, at a resolution of $8 \mathrm{~cm}^{-1}$. De-convolution, and peak extraction from the spectra of the Type IaAB diamonds was performed using Bruker OPUS software. Peak information was transferred electronically into the
Mendelssohn and Milledge (1995) Quattro-Pro spreadsheet for quantitative analysis of the diamond impurity contents. Quantitative estimations of the amount of nitrogen aggregation was assessed in the spreadsheet based on various peak ratios.

\subsection{STATISTICAL ANALYSIS}

Multivariate statistical methods permit the estimation of the variability of a certain diamond characteristic as well as to consider relationships in all the properties simultaneously. They are used in combination to reveal the underlying structure within a set of multivariate observations (Davis 1986) .

This project involves testing of various multivariate statistical techniques such as factor analysis and discriminant analysis. The aim is to find the linear combination of variables that produces the maximum difference between previously defined groups. The method establishes which variable(s) contribute(s) significantly to the discrimination between groups. Then the identified variable(s) can be used to predict group membership for new cases with a certain level of significance. Discriminant funtion analysis is similar to analysis of variance (ANOVA/MANOVA) and compares the differences between means in the multivariate space between two or more groups of data. To test the significance of the difference between multivariate means, pooled-within-group variances and covariances, the F-value (with regard to all variables) are computed and compared for all variables. The Fvalue is the multivariate equivalent of the univariate $\mathrm{T}$ test for a significant difference between two means. Furthermore the relative contribution of a certain parameter to the overall variability can be estimated. Statsoft TM (2001), Statistica 6.0 was utilised for the statistical modelling.

\subsection{Physical Characterisation}

Physical observations were made using classification schemes developed by Robinson (1979) and Otter (1989).

\subsection{ACCESS DATABASE}

The interpreted values were imported and stored in an Access database together with the categorical data. The database environment is built for retrieval and querying of data which is enhanced by the effectiveness of the database design. It enables us to determine and quantify the proportion of key features as well as identify relationships between records of data grouped by source. The database also forms the basis for selection of multivariate data sets for statistical analysis. 
Multivariate statistical techniques are being tested and applied to the multivariate data set in order to identify groups of diamonds relating to source.

\section{DATA}

A subset of 495 cases of the $\sim 2000$ records data set was selected for General Discriminant Analysis. The selection criteria was based on data availability and otherwise unbiased. The composition of the data set is 155 cases for Helam, South Africa (McKenna 2001), 95 cases for Klipspringer, South Africa (Westerlund 2000) and 245 for Misery, Ekati, Canada (Ngo 2002). The difference in number of cases has been accounted for and there is no assumption that any locality should classify on the basis of number of cases. Values of $\mathrm{N}$, $\% \mathrm{NasB}, \mathrm{H}, \mathrm{C}$ and strength and position of platelet peak as well as color and morphology are variables included in the analysis. In figure 3 the two resulting discriminant roots are plotted where root 1

\section{CONCLUSIONS}

* There are physical properties of diamonds that can be considered key features related to a certain source.

- Nitrogen content and aggregation state can be interpreted to depend on factors related to the specific environment where the diamond resided and the environment in which a diamond resides controls and modifies characteristics of the crystal over time.

* FTIR is a non-destructive, fast and cheap technique for the analysis of diamonds.

* CL images can provide useful insights to how the growth history of a diamond may vary at different localities.

* It is likely that key characteristics and FTIR measurements vary for different areas in a complex way.

* The combined variability of the parameters mentioned can be investigated with multivariate

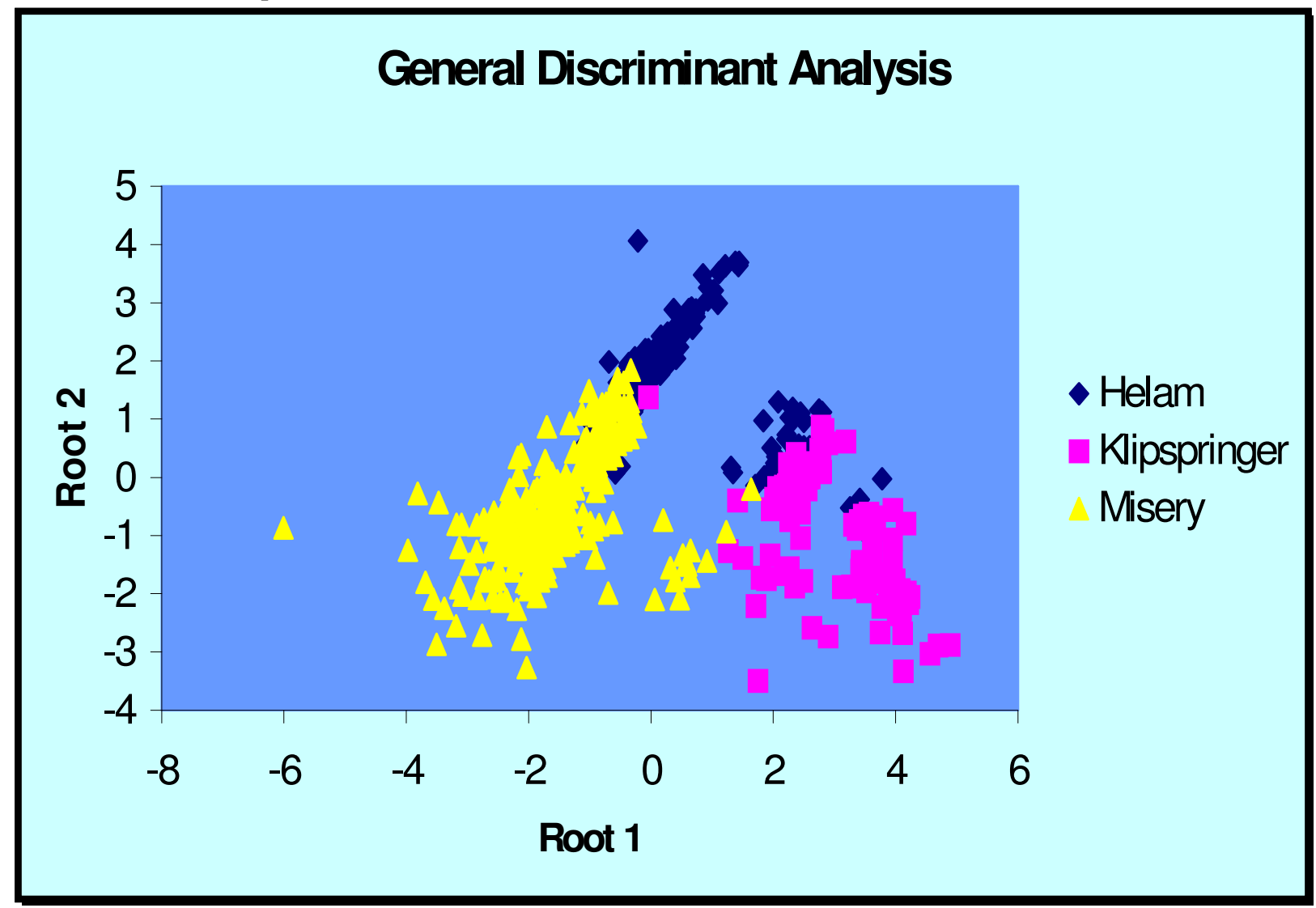

Figure 3. Result of general discriminant analysis

seems to discriminate best between Klipspringer and Misery while root 2 partly distinguishes Helam from the other two, especially Misery (Fig 3). statistics. These techniques allow us to consider changes and relationships in several properties simultaneously.

* Future work includes the acquisition of more data and further multivariate statistical analysis 
including testing of other techniques in addition to general discriminant analysis

\section{REFERENCES}

Davis, J.C., 1986. Statistics and data analysis in geology, $2^{\text {nd }}$ edition. John Wiley \& Sons.

Hawkins, D.M., 1982. Topics in applied multivariate analysis. Cambridge University Press.

Mendelssohn, M..J., Milledge, H.J., 1995. Geologically Significant Information from Routine analysis of the Mid-Infrared Spectra of Diamonds. International Geology Review. 37, 95-110.

Menzies, A.H., 2001. A detailed investigation into diamondbearing xenoliths from Newlands Kimberlite, South Africa. Ph.D. thesis, Department of Geological Sciences, University of Cape Town, South Africa.

McKenna, N., 2001. A Study of the Diamonds, Diamond inclusion minerals and other mantle minerals from the Swartruggens kimberlite, South Africa. M.Sc. thesis, Department of Geological Sciences, University of Cape Town, South Africa.

Ngo, M., 2002. Internal Report. Department of Geological Sciences, University of Cape Town, South Africa.

Otter, M.L., 1989. Diamonds and their mineral inclusions from the Sloan diatremes of the ColoradoWyoming state line Kimberlite district, North America. $\mathrm{PhD}$ thesis, Department of Geological Sciences, University of Cape Town, South Africa.

Robinson, D.N. 1979. Surface Textures and other features of Diamonds. Ph.D. thesis, Department of Geological Sciences, University of Cape Town, South Africa.

Statistica StatSoft, Inc. 2001. STATISTICA (data analysis software system), version 6 .

Westerlund, K.J. 2000. A Geochemical study of diamonds, diamond inclusion minerals and other mantle minerals from the Klipspringer kimberlites, South Africa. M.Sc. thesis, Department of Geological Sciences, University of Cape Town, South Africa.

Contact: EC Anckar, Department of Geological Sciences, University of Cape Town, Rondebosch 7701, South Africa,

E-mail: eanckar@geology.uct.ac.za 